\title{
IMPACTOS DAS AÇÕES DE EDUCAÇÃO EM SAÚDE FRENTE À PESSOA IDOSA NA ATENÇÃO PRIMÁRIA: UMA REVISÃO INTEGRATIVA DE LITERATURA
}

\author{
IMPACTS OF HEALTH EDUCATION ACTIONS TOWARDS THE \\ ELDERLY IN PRIMARY CARE: AN INTEGRATIVE LITERATURE \\ REVIEW
}

Janaína Barbosa Calixto dos Santos ${ }^{1}$ Luciene Karine Araújo dos Santos ${ }^{2}$

\begin{abstract}
RESUMO: Objetivo: identificar os impactos das ações de educação em saúde frente à pessoa idosa na atenção primária. Método: trata-se de uma Revisão Integrativa de literatura a qual utilizou artigos publicados entre 2012 a 2019, dispostos nas bases de dados da Literatura Científica e Técnica da América Latina e Caribe (LILACS) e Medical Literature Analysis and Retrieval Sistem Online (MEDLINE). Foi desenvolvido em seis etapas: 1. Identificação da questão de pesquisa (Quais os impactos das ações de educação em saúde frente à pessoa idosa na atenção primária?), 2. Definição dos critérios de inclusão e exclusão de estudos, 3. Determinação das informações a serem extraídas dos estudos selecionados/ categorização dos estudos, 4. Avaliação dos estudos incluídos na revisão integrativa de literatura, 5. Interpretação dos resultados e 6. Apresentação da revisão/síntese do conhecimento. Resultados: Diante dos critérios de inclusão e exclusão, foram designados seis artigos para compor a amostra final do estudo. Constatou-se que, as ações educativas no âmbito da atenção primária trazem impactos positivos na vida dos idosos pois promovem socialização, autocuidado, valorização e qualidade de vida, bem como estimulam o envelhecimento ativo com qualidade ressaltando o diálogo como ferramenta de extremo valor. Conclusão: É notório o impacto das ações educativas na vida dos idosos no âmbito da atenção primária, destacando-se: o empoderamento, autonomia, e boa desenvoltura no processo de envelhecimento saudável. Assim, é primordial que os profissionais de saúde continuem investindo na realização dessas práticas. Sugere-se a realização de mais estudos que possam mensurar de maneira mais acurados tais impactos.
\end{abstract}

\footnotetext{
${ }^{1}$ Graduanda em Enfermagem Bacharel pela Universidade Tiradentes - UNIT, Maceió - AL.

${ }^{2}$ Graduanda em Enfermagem Bacharel pela Universidade Tiradentes - UNIT, Maceió - AL.
} 
Palavras chave: Educação em Saúde. Idoso. Atenção Primária à Saúde.

\begin{abstract}
Objective: to identify the impacts of health education actions on the elderly in primary care. Method: it is an Integrative Literature Review which used articles published between 2012 to 2019, available in the databases of Scientific and Technical Literature of Latin America and the Caribbean (LILACS) and Medical Literature Analysis and Retrieval Sistem Online (MEDLINE). It was developed in six stages: 1. Identification of the research question (What are the impacts of health education actions against the elderly in primary care?), 2. Definition of inclusion and exclusion criteria for studies, 3. Determination ofinformation to be extracted from the selected studies / categorization of the studies, 4. Evaluation of the studies included in the integrative literature review, 5. Interpretation of the results and 6. Presentation of the review / synthesis of knowledge. Results: In view of the inclusion and exclusion criteria, six articles were designated to compose the final sample of the study. It was found that educational actions in the context of primary care bring positive impacts on the lives of the elderly because they promote socialization, selfcare, appreciation and quality of life, as well as stimulate active aging with quality, emphasizing dialogue as a tool of extreme value. Conclusion: The impact of educational actions on the lives of the elderly in the context of primary care is notable, with emphasis on: empowerment, autonomy, and good resourcefulness in the healthy aging process. Thus, it is essential that health professionals continue to invest in carrying out these practices. It is suggested to carry out more studies that can more accurately measure such impacts.
\end{abstract}

Keywords: Health Education. Old man. Primary Health Care. 


\section{INTRODUÇÃO}

Mediante um conceito mais amplo, a educação em saúde consiste em um processo dinâmico que visa mediar melhoria nas condições de saúde da população. A mesma envolve: profissionais de saúde, pacientes, gestores dentre outros. Esta prática tem se desenvolvido de maneira significativa, principalmente nos âmbitos de prevenção e promoção à saúde, nos últimos anos (SILVEIRA et al., 2015).

A educação em saúde está ligada intrinsecamente ao processo de trabalho dos profissionais da Atenção Primária à Saúde (APS) pois a partir da mesma é possível desenvolver ações educativas a fim de interferir no processo saúde-doença da população, buscando qualidade de vida, bem como autonomia individual e coletiva (MENDONÇA et al., 2017).

Evidenciam-se duas classificações para educação em saúde: a tradicional e a dialógica. A tradicional também compreendida como preventiva, se embasa no método teórico-prático e seu referencial se detém ao biologicista pois possui foco na patologia e suas intervenções. Já oformato dialógico consiste no diálogo bidirecional entre os indivíduos envolvidos, com o uso de análise e reflexões críticas sobre aspectos da realidade, procura promover planos de transformação desta, rompendo assim o modelo tradicional (MENDONÇA et al., 2017).

A partir da Constituição Federal Brasileira de 1988, foi concebida ao SUS várias competências, dentre elas encontram-se a ordenação da formação na área da saúde, deste modo as questões relacionadas à educação em saúde são peculiares ao SUS. Com intuito de efetivar e observar essa concessão, o Ministério da Saúde (MS) desenvolveu dentre outras políticas e estratégias a Política Nacional de Educação Permanente (PNEP), que visa uma prática reflexiva e transformadora durante o cotidiano no ambiente de trabalho, com vistas a adequação profissional ao contexto da população e desenvolvimento do SUS (NOGUEIRA et al., 2019).

No que tange à Atenção Primária à Saúde (APS), a Educação Permanente em Saúde (EPS) é imprescindível especialmente durante as práticas nos serviços 
de saúde, pois propõe a reestruturação da APS com aplicação dos princípios do SUS. Como principais atuantes neste cenário destacam-se os profissionais que prestam serviços de maneira integral aos usuários e familiares em todos os estágios da evolução humana, incluindo o envelhecimento populacional e assistência aos idosos (NOGUEIRA et al., 2019).

De acordo com o MS do Brasil (2020), é considerada pessoa idosa aquela que possui mais de 60 anos. Neste contexto, o índice de envelhecimento está em torno de $14,3 \%$ de toda a população brasileira este fato decorre, em geral, da melhoria dos determinantes de saúde. O MS por meio da Coordenação de Saúde da Pessoa Idosa, é responsável por implementar a Política Nacional de Saúde da Pessoa Idosa (PNSPI) normalizada pela portaria $\mathrm{n}^{\circ} 2.528$, de 19 de outubro de 2006, a qual possui dentre suas diretrizes o envelhecimento saudável e ativo, atenção integral e integrada da mesma.

Neste contexto, esta pesquisa tem como objetivo, identificar os impactos das ações de educação em saúde frente à pessoa idosa na atenção primária.

\section{MÉTODO}

Este estudo configura-se em uma revisão integrativa de literatura, a qual busca identificar os impactos das ações de educação em saúde diante da pessoa idosa na atenção primária à saúde, entre 2012 a 2019. E para tal, adotou-se a revisão integrativa de literatura de acordo com Mendes, Silveira e Galvão (2008), a fim de analisar as pesquisas relevantes, de maneira sistemática e organizada, que servirão para direcionar a pesquisa, selecionar osestudos relevantes os quais serão analisados em busca da síntese de conclusão do estudo em questão.

Para seu desenvolvimento foram seguidas as 6 etapas recomendadas por Mendes, Silveira e Galvão (2008): 1. Identificação do tema e seleção da hipótese ou questão de pesquisa para a elaboração da revisão integrativa de literatura, 2. Estabelecimento de critérios para inclusão e exclusão de estudos/ amostragem ou busca na literatura, 3. Definição das informações a serem extraídas dos 
estudos selecionados/ categorização dos estudos, 4. Avaliação dos estudos incluídos na revisão integrativa, 5. Interpretação dos resultados e 6. Apresentação da revisão/síntese do conhecimento.

Na primeira etapa, com a finalidade de guiar esta pesquisa, foi elaborada a seguinte questão norteadora: Quais os impactos das ações de educação em saúde frente à pessoa idosa na atenção primária?

Na segunda etapa, foram estabelecidos como critérios de inclusão: a) artigos publicados no período de 2012 a 2019; b) no idioma português; c) desenvolvidos no âmbito da atenção primária à saúde que respondessem à questão de pesquisa. Foram critérios de exclusão: a) estudos de revisão de literatura ou com dados secundários.

Nesta etapa foi realizada ainda a definição das estratégias de busca as quais utilizaram Descritores de Ciências da Saúde (DeCS) e palavras-chave: educação em saúde; idoso e atenção primária à saúde. Para tal, foi utilizado as seguintes estratégias de busca: educação em saúde AND idoso AND atenção primária à saúde; 2) idoso AND educação em saúde AND atenção primária à saúde.

A busca dos artigos nas bases de dados foi dada através da busca no portal da Biblioteca Virtual de Saúde (BVS), sendo selecionadas as bases de dados Literatura Científicae Técnica da América Latina e Caribe (LILACS), a qual resultou em 54 artigos e Medical Literature Analysis and Retrieval Sistem Online (MEDLINE) que compôs 63 artigos, no período de fevereiro de 2020.

Os artigos selecionados foram minuciosamente analisados. Por fim, foram excluídos artigos que se encontravam em duplicidade nas bases de dados.

\section{RESULTADOS}

Após abordagem inicial nas bases de dados, foram identificados 117 artigos dos quais após leitura dos títulos restaram 36, prosseguindo com a leitura dos resumos permaneceram 6 artigo; após leitura destes na íntegra os 06 artigos permaneceram e compuseram a amostra final do estudo. 
A caracterização dos artigos que compuseram a amostra encontra-se disposta no Quadro 2. Em relação ao delineamento do estudo houve predomínio de estudos qualitativos (02) e derevisão integrativa (02), a maioria foi publicado no ano de 2018 (02).

Quadro 2. Síntese dos estudos primários relacionados aos temas.

\begin{tabular}{|c|c|c|c|}
\hline $\begin{array}{c}\text { AUTOR (ES)/ } \\
\text { ANO DE } \\
\text { PUBLICAÇÃO }\end{array}$ & OBJETIVO & METODOLOGIA & $\begin{array}{l}\text { RESULTADOS } \\
\text { PRINCIPAIS }\end{array}$ \\
\hline $\begin{array}{l}\text { 1. NOGUEIRA, et } \\
\text { al/2019 }\end{array}$ & \begin{tabular}{|lr} 
Desvelar & as \\
práticas & de \\
Educação & \\
Permanente & em \\
Saúde & (EPS) \\
desenvolvida & pelo \\
Núcleo de Apoio à \\
Saúde da Família \\
na atenção ao \\
idoso.
\end{tabular} & $\begin{array}{l}\text { Estudo qualitativo, } \\
\text { exploratório- } \\
\text { descriti vo. }\end{array}$ & $\begin{array}{l}\text { As práticas de EPS ao } \\
\text { idoso ocorrem durante } \\
\text { os momentos de } \\
\text { discussão de casos, no } \\
\text { matricialmente, nas } \\
\text { visitas domiciliares, nos } \\
\text { grupos operativos e no } \\
\text { cotidiano do trabalho de } \\
\text { modo informal. }\end{array}$ \\
\hline $\begin{array}{l}\text { 2. SILVEIRA, } \\
\text { al/2015 }\end{array}$ & $\begin{array}{l}\text { Avaliar a produção } \\
\text { científica } \\
\text { relacionada } \\
\text { educação } \\
\text { saúde para } \\
\text { no Brasil. }\end{array}$ & $\begin{array}{l}\text { Estudo de revisão } \\
\text { integrativa }\end{array}$ & $\begin{array}{l}\text { As oficinas } \\
\text { contribuíramde modo } \\
\text { inovador para estimular } \\
\text { a participação dos idosos, } \\
\text { promovendo a interação e } \\
\text { o convívio dos mesmos, } \\
\text { enquanto assumiram o } \\
\text { papel de agentes ativos } \\
\text { no processo de } \\
\text { aprendizagem e de fazer } \\
\text { saúde. }\end{array}$ \\
\hline $\begin{array}{l}\text { 3. CARVALHO, et } \\
\text { al/2018 }\end{array}$ & $\begin{array}{|lr|}\mid \text { Identificar } & \text { na } \\
\text { literatura } & \text { a } \\
\text { produção } & \text { científica } \\
\text { sobre } & \text { as } \\
\text { intervenções } & \\
\text { educativas } & \\
\text { utilizadas } & \text { por } \\
\text { enfermeiros } & \text { na } \\
\text { promoção } & \text { da } \\
\text { saúde do idoso. } & \end{array}$ & $\begin{array}{l}\text { Revisão integrativa } \\
\text { de literatura }\end{array}$ & $\begin{array}{l}\text { Empoderamento } \\
\text { autonomia. Mudanças } \\
\text { positivas para todos os } \\
\text { atores envolvidos no } \\
\text { processo educativo. }\end{array}$ \\
\hline
\end{tabular}




\begin{tabular}{|c|c|c|c|}
\hline $\begin{array}{l}\text { 4. FILHO, } \\
\text { al/2018 }\end{array}$ & 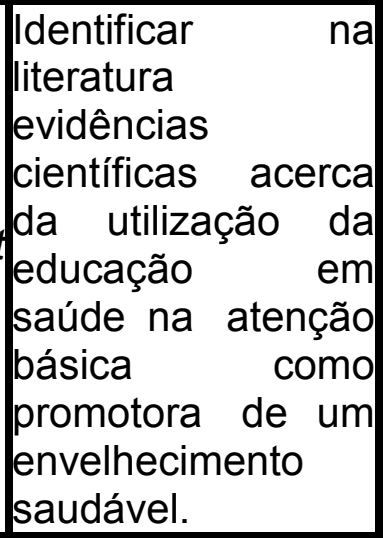 & $\begin{array}{l}\text { Revisão } \\
\text { bibliográfica } \\
\text { literatura } \\
\text { literatura }\end{array}$ & da $\mid \begin{array}{lrr}\text { As ações de educação } \\
\text { em saúder são } \\
\text { reconhecidas remo } \\
\text { essenciais para o o } \\
\text { cuidado destinado à } \\
\text { pessoa idosa, no sentido } \\
\text { de garantir aos sujeitos } \\
\text { empoderamento } \\
\text { relacionado a sua saúde. }\end{array}$ \\
\hline $\begin{array}{l}\text { 5. MENDONÇA, } \\
\text { et al/2017 }\end{array}$ & $\begin{array}{|lr|}\text { Avaliar } & 0 \\
\text { desenvolvimento } & \text { e } \\
\text { implementação } & \text { de } \\
\text { uma ação } & \text { de } \\
\text { educação } & \\
\text { permanente. } & \\
\end{array}$ & $\begin{array}{l}\text { Estudo } \\
\text { abordagem } \\
\text { qualitativo- } \\
\text { quantitativa }\end{array}$ & de $\begin{array}{l}\text { O grupo de educação } \\
\text { em saúde promoveu } \\
\text { empoderamento, } \\
\text { qualidade de vida e } \\
\text { socialização para os } \\
\text { idosos. }\end{array}$ \\
\hline 6. LUZ, etal/2012 & $\begin{array}{l}\text { Avaliar as ações } \\
\text { estratégicas } \\
\text { atenção à } \\
\text { do idosaúde } \\
\text { Unidades } \\
\text { de Saúde(Uásicas } \\
\text { Teresina-PI. }\end{array}$ & $\begin{array}{l}\text { Estudo } \\
\text { observacional } \\
\text { transversal }\end{array}$ & \begin{tabular}{|lr} 
A maioria dos & idosos \\
$(70 \%)$ afirmaram & que os \\
profissionais de & saúde \\
preocupam-se & em \\
executar as & ações \\
coletivas, de promoção à \\
saúde, racialização \\
dentreoutros.
\end{tabular} \\
\hline
\end{tabular}

Fonte: Elaborado pelos autores.

\section{SÍNTESE DA REVISÃO INTEGRATIVA DE LITERATURA}

Conforme análise dos resultados dos estudos selecionados para esta pesquisa dispostos no quadro sinóptico 2, a educação em saúde traz impactos relevantemente significativos paraa vida dos idosos.

A EPS é entendida como uma ferramenta educativa que proporciona uma discussão crítica das ações realizadas nos serviços de saúde envolvendo os profissionais e idosos, alicerçando por essência a abordagem dialógica que por consequência tornam as ações educativas mais eficientes e abrangentes, alcançando os objetivos e trazendo impactos positivos na vida da população idosa (NOGUEIRA et al., 2019). 
O diálogo é um importante veículo para o cuidado quando bem utilizado na educação em saúde, pois estimula a participação dos idosos propiciando respeito ao outro, troca de experiências, interação e construção de vínculos. Assim, o diálogo promove impactos positivos para esses indivíduos, tornando-os mais ativos no processo de aprendizagem e no desenvolver da saúde (SILVEIRA et al., 2015).

Neste sentido através do diálogo é possível implementar a educação em saúde seja ela grupal ou individual. Nestas interações há troca de conhecimentos e experiências que visam à promoção da saúde, com incentivo à prática de exercício físico e dieta adequada, dentre outros fatores que propiciam impactos relevantemente positivos para desenvolvimento de um envelhecimento saudável. Neste contexto, a comunicação é uma ferramenta essencial para se educar em saúde, pois traz o indivíduo para o centro do autocuidado, promovendo saúde, autonomia e empoderamento com vistas na mudança de comportamento e quebra do método tradicional onde se enfatiza consultas e exames (CARVALHO et al., 2018).

Observa-se que as práticas educativas são recursos positivos, pois impactam e modificam positivamente o comportamento adquirido no cotidiano, ressaltando a alimentação saudável, e aumento da prática de atividade física. Bem como, na melhoria da saúde mental uma vez que oportunizam momentos de socialização e melhora da autoestima dos idosos. Vale ressaltar que, as práticas educativas devem ser focadas nos pacientes verificando suas necessidades e angústias para então, programar e executar as ações educativas. E ainda trazerà tona a necessidade de os profissionais estarem qualificados para desenvolverem tais ações (FILHO et al., 2018).

As ações de educação em saúde são um desafio entre os profissionais e gestores, pois estes, na maioria das vezes tiveram em suas formações profissionais o acesso ao modelo tradicional. Outro aspecto é que a falta de recursos adequados muitas vezes torna o modelo tradicional fácil de ser aplicado (MENDONÇA et al., 2017).

Entretanto, as ações de educação em saúde vinculadas ao grupo de idosos impactam o cotidiano destes, pois promovem socialização, autocuidado, valorização e qualidade de vida, bem como estimulam o envelhecimento ativo e com qualidade. 
Quando essas práticas são realizadas com olhar amplo e abertas para alterações juntamente com diversos materiais, abrem espaços para o diálogo favorecendo a aproximação e conhecimento dos indivíduos, tornando as práticas efetivas e prazerosas (MENDONÇA et al., 2017).

Diante de um questionário aplicado, observa-se que $70 \%$ das respostas de uma pergunta remetem aceitação das ações de educação em saúde, a qual reflete impactos na melhoria da qualidade de vida desses idosos, promoção da saúde e socialização, a qual faz cumprir a PNSPI bem como o Estatuto do Idoso. (LUZ et al., 2012).

\section{CONCLUSÃO}

O objetivo do estudo foi alcançado, pois foi possível identificar os impactos das ações de educação em saúde frente à pessoa idosa na atenção primária.

Dentre esses impactos destacaram-se: melhoria na qualidade de vida, socialização, autocuidado, envelhecimento ativo e saudável, troca de conhecimentos/experiências, respeito ao outro, promoção a saúde, bem como melhor interação entre os profissionais e o público idoso. Deste modo, torna-se efetiva a PNSPI propiciando um envelhecimento ativo e com qualidade.

Ressalta-se que é de suma importância que os profissionais de saúde detenham o conhecimento sobre os impactos das ações de educação em saúde mediante ao público idoso, e que a partir disso possam embasar suas práticas a fim de ofertar um cuidado com excelência. Sugere-se a realização de mais estudos que possam mensurar de maneira mais acurados tais impactos. 


\section{REFERÊNCIAS BIBLIOGRÁFICAS}

BRASIL. Ministério da Saúde. Saúde da pessoa idosa: prevenção e promoção à saúde integral: [Ministério da Saúde], 2020.

CARVALHO, K. M.; SILVA, C. R. D. T.; FIGUEIREDO, M. L. F.; NOGUEIRA, L. T.; ANDRADE, E. M. L. R. Intervenções educativas para promoção da saúde do idoso: revisão integrativa. Acta paul. enferm., São Paulo , v. 31, n. 4, p. 446-454, July 2018 . Disponívelem: <http://http://www.scielo.br/scielo.php?script=sci_arttext\&pid=S0103-21002018000400446>. acesso em: 15 Fev. 2020.

FILHO, F. J. R. L.; SILVA, I. G. B.; LIMA, N. K. G.; PONTES, M. M.; SOUSA, Y. P.; OLIVEIRA, T. W. N. AÇÕES DE EDUCAÇÃO EM SAÚDE PARA IDOSOS NA ATENÇÃO BÁSICA: REVISÃO DE LITERATURA. Revista Contexto \& Saúde, v. 18, n.35, p. 34-41, 20 dez. 2018.2 Disponivel em: <https://www.revistas.unijui.edu.br/index.php/contextoesaude/article/view/7481>.

LUZ, L. A. DA; FUTINO, M. I.; LUZ, E. A. DA; MARTINS, G. M.; BARBOSA, E. P. M.; ROCHA, L. DE M. Avaliação das ações estratégicas na atenção à saúde do idoso em Unidades Básicas de Saúde de Teresina-PI. Revista Brasileira de Medicina de Família e Comunidade, v. $7, \quad$ n. 22, p. 20-26, 11 fev. 2012. Disponivel em: <https://www.rbmfc.org.br/rbmfc/article/view/379/453>. Acesso em: 10 fev. 2020.

MENDES, K.S; SILVEIRA, R. C. C. P; GALVÃO, C.M. Revisão Integrativa: Método de pesquisa para a incorporação de evidência na saúde e na enfermagem. Texto Contexto Enferm., Florianópolis - SC, v.17, n.4, p. 758 - 764, Out - Dez. 2008. Disponível em: < http://www.scielo.br/pdf/tce/v17n4/18.pdf. Acesso em : 09 de fevereiro de 2020.

MENDONÇA, F. T. N. F.; SANTOS, A. S.; BUSO, A. L. Z.; MALAQUIAS, B. S. S. Educação em saúde com idosos: pesquisa-ação com profissionais da atenção primária. Rev. Bras. Enferm., Brasília , v. 70, n. 4, p. 792-799, Aug. 2017. Disponível em: <http://http://www.scielo.br/scielo.php?pid=S0034-71672017000400792\&script=sci_arttext\& tlng=pt>. acesso em: 15 Fev. 2020.

NOGUEIRA, I. S.; ACIOLI, S.; CARREIRA, L.; BALDISSERA, V. D. A. Atenção ao idoso: práticas de educação permanente do Núcleo de Apoio à Saúde da Família. Rev. esc. enferm. USP, São Paulo, v. 53, 2019 Disponivel em: <http://http://www.scielo.br/scielo.php?pid=S008062342019000100468\&script=sci_arttext>. acesso em: 15 Fev. 2020.

SILVEIRA, R. E., MENDONÇA, F. T. N. F., SANTOS, A. S. Y.; FILIPE, E. M. V. Estratégias de educação em saúde para idosos: experiências e desafios. Cultura de los Cuidados. (Edición digital), v. 19, n. 42, Junho 2015. Disponivel em: <https://rua.ua.es/dspace/bitstream/10045/49338/1/Cultura-Cuidados_42_14.pdf> acesso em: 10 fev. 2020. 